\title{
Umbral adaptivo para sistemas de comunicaciones ópticas inalámbricas por medio de algoritmos de agrupamiento
}

\author{
Juan de Dios Sánchez López, Juan Iván Nieto Hipólito, \\ Mabel Vázquez Briseño, Victor R. Velázque M. \\ Universidad Autónoma de Baja California, Facultad de Ingeniería Arquitectura y Diseño, \\ Baja California, México \\ \{jddios, jnieto, mabel.vazquez, vvmejia\}@uabc.edu.mx
}

\begin{abstract}
Resumen. Sistemas basados en modulación de intensidad y detección directa tipo OOK (Conmutación de Encendido -Apagado, o del inglés OOK: On-Off Keying). Son de uso extendido en las redes fotónicas actuales debido a su sencillez y robustez pero no son los más eficientes en términos del uso del espectro. Sistemas de modulación de intensidad multi-simbólicos como M-PAM (del inglés: M-ary Pulse Amplitud Modulation) los cuales presentan alta eficiencia espectral han sido vistos como alternativa a formatos de modulación tradicionales, sin embargo son más complejos y en ciertos casos requieren de un umbral adaptivo en aplicaciones de comunicaciones ópticas inalámbricas (COI). Se presenta en este trabajo un método de selección de umbral que utiliza algoritmos de agrupamiento basado en las K-medias y el desempeño del uso de este umbral adaptivo en sistemas COI con M-PAM, por medio del simulador Matlab®
\end{abstract}

Palabras clave: K-medias, comunicaciones ópticas inalámbricas, umbral adaptivo.

\section{Introducción}

En las décadas pasadas se ha incrementado en interés en los sistemas de comunicaciones ópticas de espacio libre. Si bien son un nicho de aplicación debido a las ventajas, tales como costos relativamente bajos de instalación, no están sujetos a regulación por el uso del espectro radioeléctrico, anchos de banda comparables a los de los sistemas ópticos de fibra óptica, entre otras, pero presentan alta susceptibilidad a las condiciones atmosféricas, tales como la niebla, aerosoles, e inclusive en condiciones de cielo claro son afectados por la turbulencia óptica atmosférica $[1,2,3]$. Este fenómeno es uno de los que más restringe debido a la destrucción de la coherencia temporal como espacial del haz láser ocasionando variaciones de fase y amplitud (y por tanto de intensidad) de la señal trasmitida. La turbulencia atmosférica es un fenómeno ocasionado por el calentamiento desigual de masas de aire, los que producen variaciones del índice de refracción a lo largo de la trayectoria de un enlace de COI, 
produciendo variaciones de intensidad ocasionando degradación del desempeño del sistema e inclusive caída del enlace.

Este campo de estudio de las telecomunicaciones está en pleno desarrollo y se ha realizado diversos estudios e infinidad de propuestas para resolver los problemas propios de estos sistemas como son su baja disponibilidad si se le compara con otros sistemas [3, 4, 5, 7, 8]. En [5] se analiza la utilización de MPAM para aplicaciones de Comunicaciones con Luz Visible (CLV) utilizando en combinación con esquemas de modulación múltiples frecuencias ortogonales (MMFO o en inglés: Ortogonal Frequency Multiple Modulation, OFDM). El uso de señales PAM (Modulación por Amplitud de Pulso o PAM del inglés Pulse Amplitude Modulation) multisímbolicas incrementan la razón bit/s/Hz en comparación de señales que emplean Conmutación de Encendido -Apagado (OOK: On-Off Keying).

En la Fig. 1 se muestra el umbral para el caso de una señal binaria, en la cual la probabilidad de detección para el símbolo "0" y "1" son descritos por la función densidad de probabilidad que describe el canal para los símbolos binarios. Para el caso de MPAM con $M=4$ se requieren de tres umbrales para la decisión de los símbolos transmitidos (dos bits por símbolo para este caso). El umbral adecuado u óptimo puede ser determinado por medio de algunas de las diferentes técnicas de optimización, el cual es elegido de acuerdo a las condiciones del canal y tomando nuevos valores tan pronto se presenten nuevas condiciones en la señal recibida. Sin embargo las técnicas de optimización pueden requerir de evaluaciones de expresiones matemáticas que en el caso de los canales ópticos atmosféricos involucran funciones complicadas tales como la integración numérica de las funciones densidad de probabilidad ( f.d.p,) lognormal, gamma-gamma, etc. que caracterizan este tipo de canales, lo que hace necesario el uso de gran cantidad de recurso computacional y de tiempo de procesamiento. Este trabajo presenta una forma de determinar estos umbrales para ser utilizados en la detección de señales multisimbólicas sin requerir cantidades excesivas de recursos de cómputo por medio del uso del algoritmo de agrupamiento K-medias.

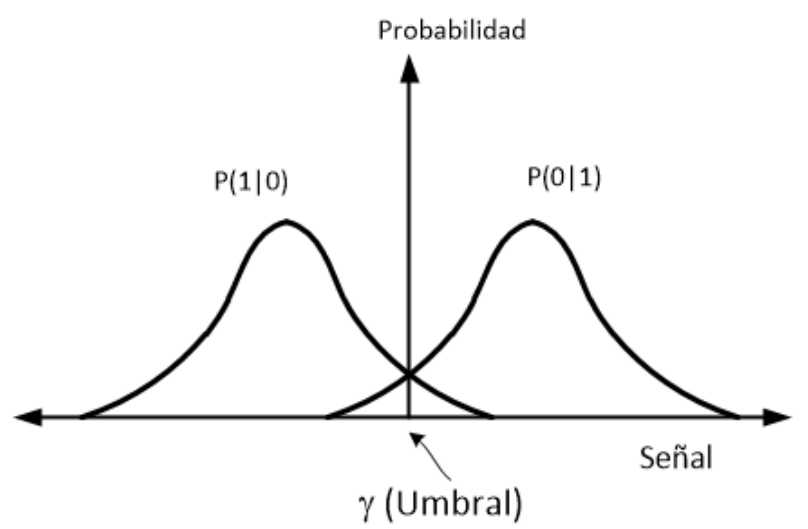

Fig. 1. Funciones de densidad de probabilidad gaussianas para los bits "0" y "1.

En la Fig. 2 se muestra la forma en que opera el umbral fijo $\gamma_{F}$ y para un umbral adaptivo $\gamma_{A}$ para una señal que lleva información binaria en los cambios de intensidad. En el caso de que la señal binaria este por encima de este, será considerada como un "1" binario o un "0" cuando se encuentre debajo de este. Errores ocurren cuando la 
Umbral adaptivo para sistemas de comunicaciones ópticas inalámbricas por medio de algoritmos ...

señal correspondiente al "1" afectada por el desvanecimiento cae por debajo del umbral detectando erróneamente como un " 0 " y el caso contrario ocurre cuando el ruido excede el umbral detectándolo como un "1", lo que es conocido como falsa alarma. Lo anterior puede ser reducido midiendo la señal y seleccionando el mejor umbral, minimizando los errores debido al uso de un umbral fijo en señales que experimentan desvanecimiento.

(a)

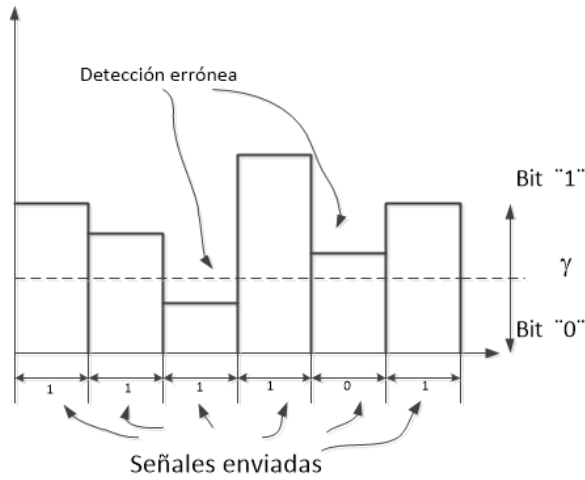

(b)

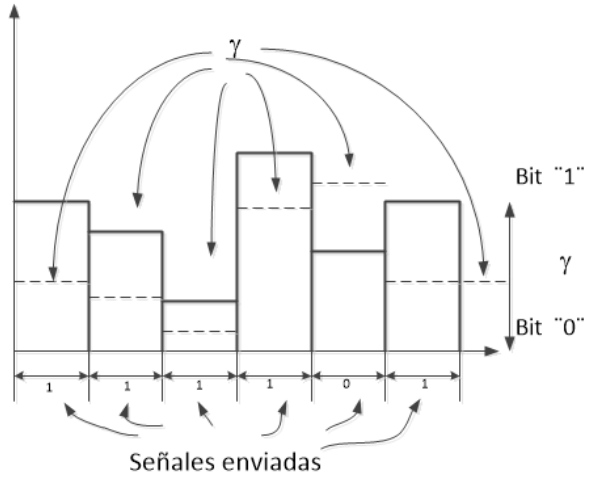

Fig. 2. Señales binarias y umbral a) Detección con umbral fijo. b) Detección con umbral adaptivo.

Este trabajo tiene como objetivo el mostrar un método de obtención del umbral adaptivo para señales MASK (o MPAM) por medio de algoritmos de agrupamiento, específicamente K-medias. Se considera que la turbulencia óptica atmosférica está dentro del régimen de turbulencia débil, (varianza de Rytov, $\sigma_{R}^{2}<1$ ). Por la utilización de estas funciones y diseñando un algoritmo para estas condiciones, se obtiene el umbral óptimo. El desempeño del sistema es evaluado por simulación, en los cuales el algoritmo estima el umbral adecuado (sub-óptimo) para las condiciones del canal en un momento determinado.

El resto del documento es organizado como sigue: En la sección 2 se describe el modelo del sistema COI basado en modulación de intensidad y detección directa con modulación OOK. y MPAM. En la sección 3 se presenta un resumen del método de detección de umbral basado en la técnica de agrupamiento en "K-means" o K-medias y en la sección 4 se presentan la implementación, los criterios utilizados para la misma y la simulación del sistema. En la sección 5 se presentan los resultados obtenidos y en la 6 se presentan algunas conclusiones y observaciones del trabajo.

\section{Modelo del sistema de comunicaciones ópticas inalámbricas}

Uno de los tipos más representativos de los sistemas COI es el que utiliza la modulación de intensidad (MI) y esta intensidad es convertida en una corriente eléctrica en el receptor por medio de un foto-receptor, (en los sistemas prácticos un diodo PIN o un diodo fotodiodo de avalancha) lo que es conocida como detección directa (DD). En este tipo de sistemas, la intensidad es modulada en la forma de conmutación de apagado 
- encendido (OOK de las siglas en inglés de On-Off Keying). En estos receptores, el campo eléctrico de la luz recibida $E_{s}$, es convertido en el receptor directamente a corriente eléctrica por medio de un foto-receptor, tal como se explicó líneas arriba. La fotocorriente es directamente proporcional al cuadrado de $E_{s}$ y por lo tanto a la potencia óptica recibida $P_{r}$, esto es:

$$
i(t)=\frac{e \eta}{h v} E_{s}^{2}(t)=\mathcal{R} P_{r}(t)
$$

donde $e$ es la carga del electrón, $\eta$ es la eficiencia cuántica, $h$ es la constante de Planck, $v$ es la frecuencia óptica. El diagrama de bloques básico del sistema se muestra en la Fig. 3

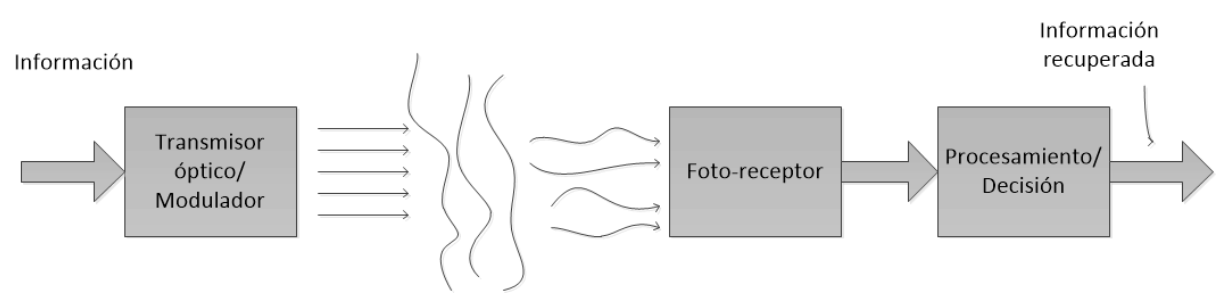

Fig. 3. Sistema de comunicaciones ópticas inalámbricas atmosférico.

Otra medida de la turbulencia es la varianza de Rytov, la cual relaciona la constante de estructura del índice de refracción, $C_{n}^{2}$ con la trayectoria del haz a través de la siguiente ecuación [6]:

$$
\sigma_{R}^{2}=1.23 C_{n}^{2} k^{7 / 6} L^{11 / 6}
$$

con $k=2 \pi / \lambda$ en donde $\lambda$ es la longitud de onda y $L$ es la distancia de la trayectoria del haz.

\subsection{Umbral adaptivo para señales 4PAM}

En los sistemas de comunicaciones ópticas en el espacio libre, debido a lo impredecible del canal óptico atmosférico es común utilizar señales que ofrezcan robustez ante condiciones como el desvanecimiento y la cintilación, tales como las señales OOK o MPPM [6]. Sin embargo en los sistemas de comunicaciones de fibra óptica, la utilización de señales PAM multi-simbólicas (MPAM) es una campo en plena actividad y desarrollo, las cuales presentan una mayor eficiencia del uso del ancho de banda[1], [2] . Una señal MPAM puede ser representada como:

$$
S_{P A M}(t)=A_{m} g(t) \operatorname{sen}\left(2 \pi f_{c} t\right), \quad m=1,2, \ldots \ldots, M
$$

donde $A_{m}$, es una de la M posibles amplitudes de la señal PAM, $g(t)$ es un pulso de amplitud unitaria, $f_{c}$ es la frecuencia de la señal portadora y $\mathrm{M}$ es la cantidad de símbolos que pueden ser representados por la señal. En la Fig. 4 se representa gráficamente la envolvente de la señal. Esta señal lleva por cada amplitud dos bits, $(\mathrm{M}=4 \mathrm{y} \mathrm{k}=2)$ y el receptor deberá de convertir estas amplitudes de las señales en una 
Umbral adaptivo para sistemas de comunicaciones ópticas inalámbricas por medio de algoritmos ...

palabra de dos bits, lo que requiere una adecuada cuantización de la señal. En el caso de canales inalámbricos, debido a la cintilación, la señal recibida experimentará variaciones en su amplitud, por lo que se requiere un continuo ajuste los niveles de cuantización o de umbrales que permitan al receptor una correcta decisión y mantenga la tasa de error de bit en niveles aceptables.

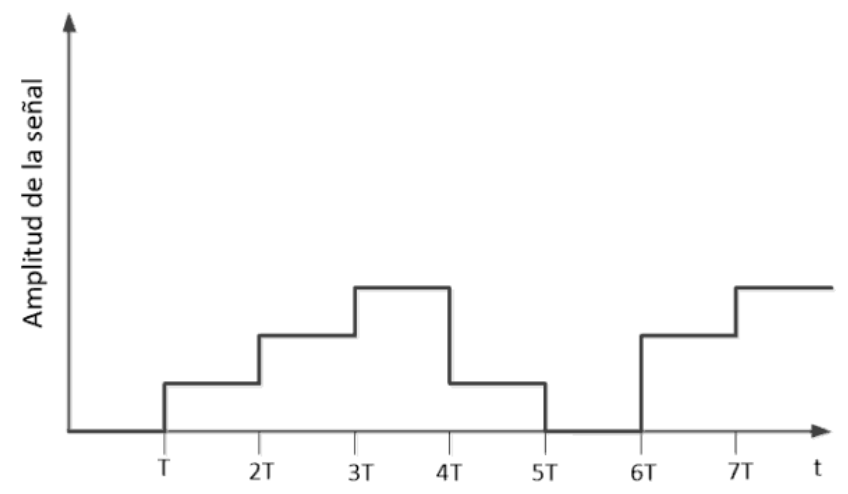

Fig 4. Envolvente de señal MPAM $(M=4)$.

\section{Método de detección del umbral}

La transmisión de palabras (grupos de bits) o vectores puede ser considerada como un problema de cuantización [9]. Además, de los fundamentos básicos de la teoría de la tasa de distorsión, se conoce que la cuantización vectorial presenta un mejor desempeño que la cuantización de escalares debido a que se hace uso de la dependencia conjuta de las muestras. Sin embargo esta técnica requiere que la función densidad de probabilidad conjunta del vector de los componentes de las amplitudes, $\mathrm{X}=[\mathrm{x} 1, . ., \mathrm{xn}]$ sea conocida, lo cual es difícil de obtener en la práctica.

Algoritmos de agrupamiento como K-media pueden ser utilizados para esto, pero debido a la velocidad de los datos transmitidos no son adecuados, pero si puede ser utilizado para la determinación de los umbrales requeridos por los subsistemas de decisión. La determinación de estos $\mathrm{M}$ diferentes niveles de umbral pueden obtenerse de la señal eléctrica foto-detectada [4] y debido a que el tiempo de correlación de la turbulencia óptica o cintilación es del orden de las decenas de milisegundos, la determinación de los umbrales requeridos pueden ser obtenidos por sistemas de procesamiento de señales digitales disponibles comercialmente tales como los arreglos de compuertas lógicas programables (FPGAS de las siglas en inglés Field Programmable Gate Array), en los cuales se ejecutaría este algoritmo.

El algoritmo de agrupamiento K-medias se basa en la obtención del cuadrado de la mínima distancia Euclideana [9, 10, 11, 12], entre el grupo de datos y el centro del agrupamiento (centroide) $\overline{\mathbf{X}}_{k}(i)$ :

$$
\bar{X}_{i}(i)=\frac{1}{M_{k}} \sum_{X \in C_{k}} X(m) \quad 1 \leq k \leq L,
$$


donde $C_{k}$ es el k-esimo agrupamiento o celda, $\mathrm{M}$ es el número de vectores de entrenamiento y L es el número de agrupamientos máximo.

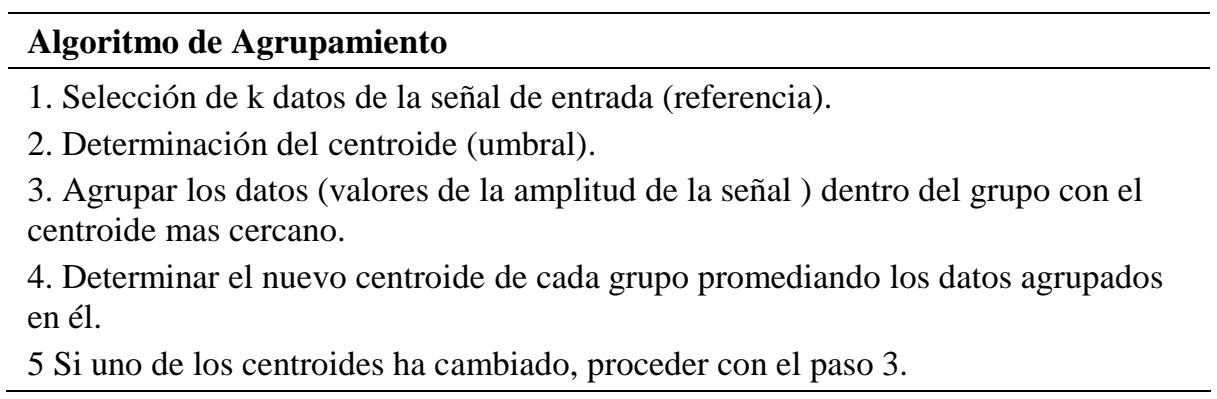

\section{Evaluación del algoritmo y experimento}

La evaluación de la utilidad del algoritmo se realizó por medio de la obtención de los valores de los umbrales para una señal 4PAM afectada por fluctuaciones similares a la ocasionada por la turbulencia atmosférica u otro fenómeno que genere desvanecimiento. La señal que emula la turbulencia se obtuvo por medio de un generador de turbulencias atmosféricas y capturadas por medio de un osciloscopio de captura y digitalización, la cual presenta una estadística con contorno similar a una f.d.p. lognormal [6]. La señal de datos modulada es obtenida de un generador de datos pseudo-aleatorios y éstos modulados por medio utilizando un generador de señales de señales arbitrarias. En este caso se utilizó $\sigma_{R}^{2}=0.1$ y una potencia de señal óptica recibida de $-30 \mathrm{dbm}$ considerando una constate de conversión de potencia óptica a eléctrica $\eta=0.3$. En la Fig. 5 se muestra el diagrama del sistema simulado en Matlab

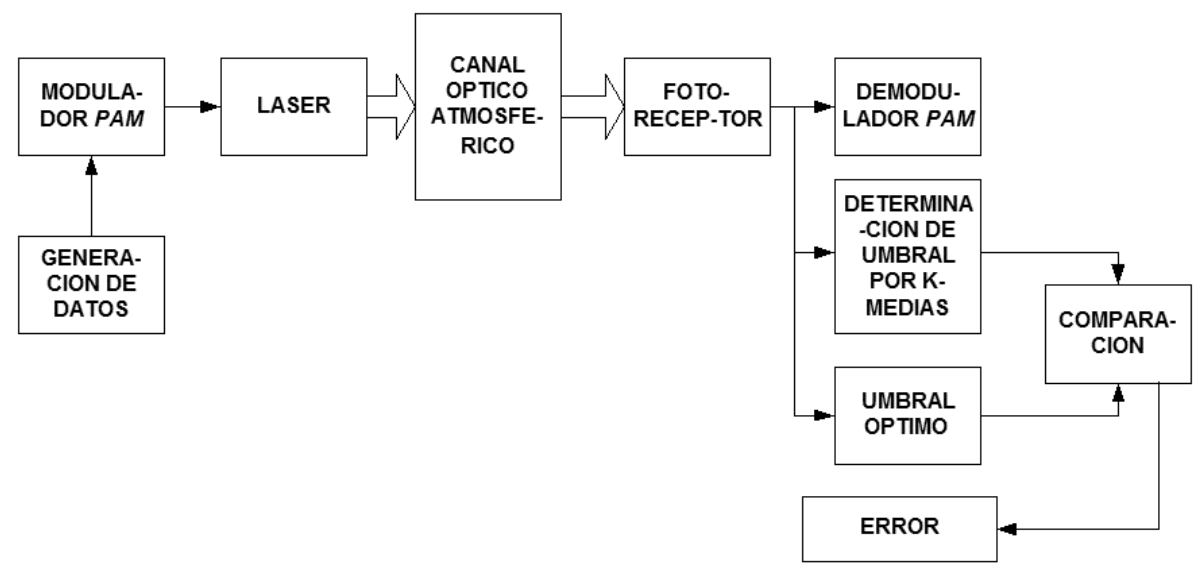

Fig. 5. Diagrama de bloques del sistema simulado.

El algoritmo determina los valores de umbral a partir del valor medio entro los centroide contiguos a partir de varias muestras de los preámbulos de cada paquete (frame) de información y actualizando cada cierto tiempo. Esto es posible debido a que el proceso lento en relación de la tasa de transmisión de bit y de esta manera establecer 
Umbral adaptivo para sistemas de comunicaciones ópticas inalámbricas por medio de algoritmos ...

en forma dinámica los nuevos umbrales. En la Fig. 6 se muestra el diagrama de constelación en el cual se muestra la determinación del centroide a partir de las muestras de la señal recibida.

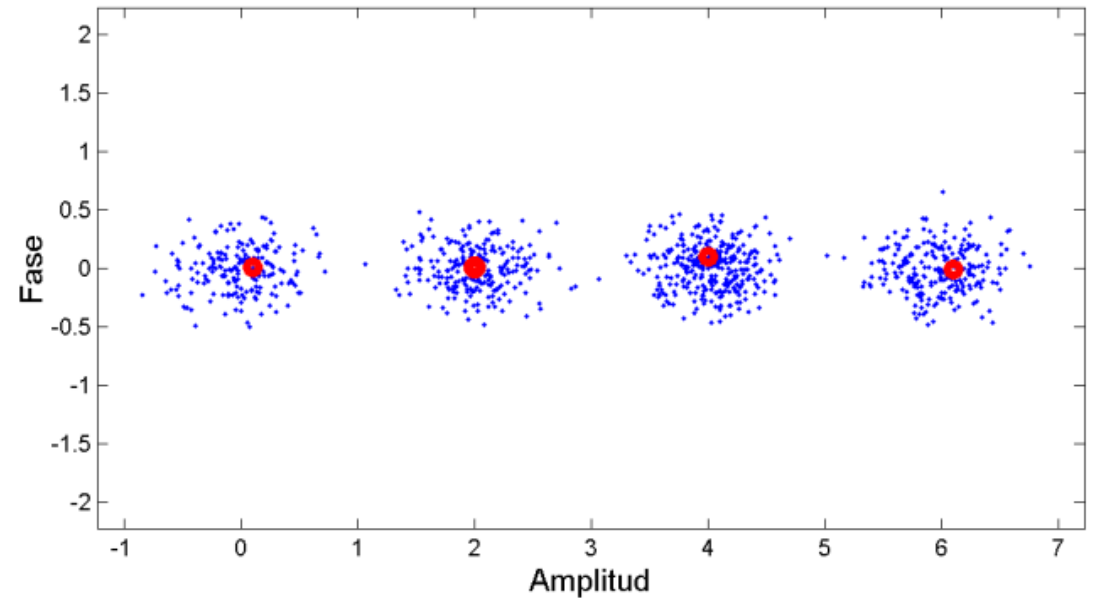

Fig. 6. Determinación de los centroides a partir de las muestras de la señal recibida.

En la Fig. 7 se muestra el error obtenido entre el umbral ideal y el establecido a partir de las mediciones y actualizado cada $10^{6}$ bits. El error puede ser reducido actualizando con periodos menores, pudiendo ser tomado a partir de cada preámbulo del paquete de información.

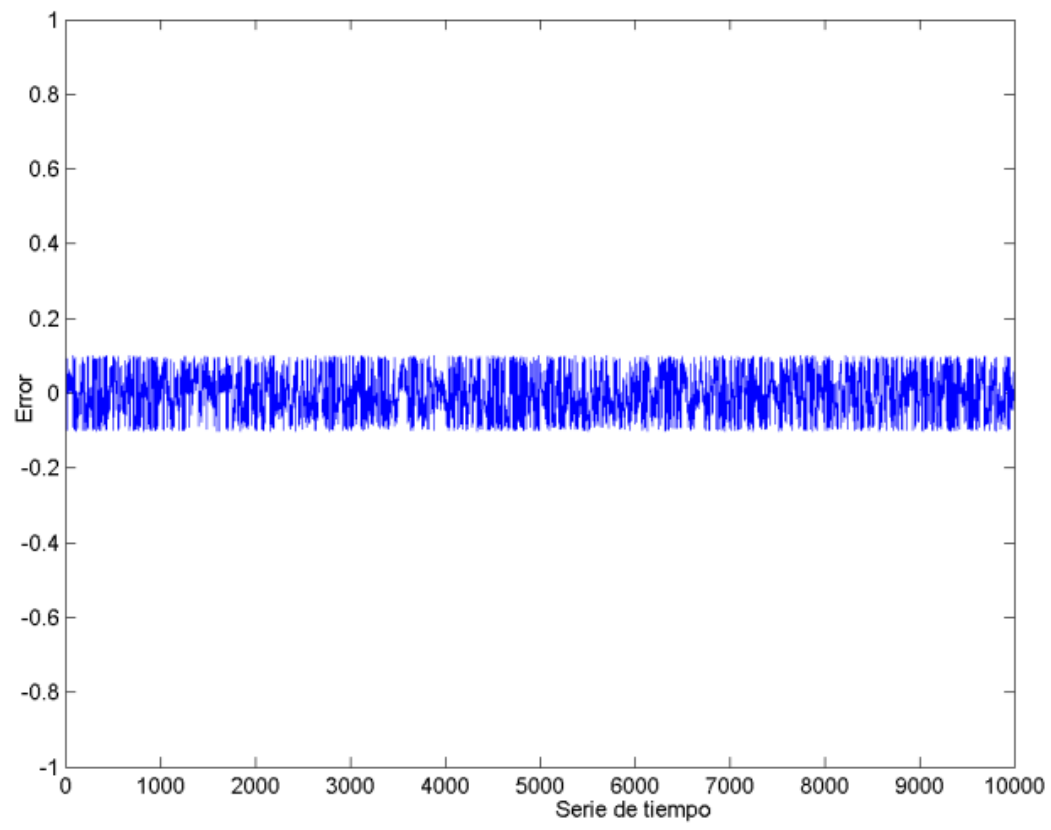

Fig. 7. Error obtenido entre el umbral ideal y el establecido. 


\section{Conclusiones y discusión}

En este trabajo se desarrolló un sistema basado en las K-medias para la determinación de los umbrales requeridos para señales M-PAM, se simuló la estructura del sistema de comunicaciones para su simulación para la evaluación por medio del Matlab. En este trabajo específico 4-PAM fue considerado y evaluado. Debido a la naturaleza del problema, no se utiliza propiamente el algoritmo para agrupar sino para determinar los centroides dado las muestras de la señal recibida la cual tiende a fluctuar ampliamente pero de forma lenta dado el proceso. Si bien el canal óptico inalámbrico puede ser muy restrictivo, puede ser considerado para la aplicación en sistemas de comunicaciones en luz visible o en sistemas de fibra óptica. El algoritmo es capaz de determinar los centroides y los umbrales requeridos para señales fluctuantes debido al desvanecimiento.

\section{Referencias}

1. Han, L., Wang, Q., Shida, K.: Outage probability of free space optical communication over atmosphere turbulence. WASE International Conference on Information Engineering, pp. 127-130, IEEE Press, China (2010)

2. Khallaf, H.S., Morra, A.E., Shalaby, M.H., Kawasaki, Z.: Performance analysis of free-space optics systems adopting multi-pulse PPM techniques in gamma-gamma channels for thermal noise limited systems. ICTON (2013)

3. Mukai, R., Arabshahi, P., Yan, T.Y.: An adaptative threshold and channel parameter estimator for deep space optical communications. IEEE, pp. 50-54, USA (2001)

4. Wang, Z., Zhong, W.D., Yu, C.: Dynamic decision threshold and adaptive coherent detection in FSO communication system. IEEE, Singapore, (2011)

5. Shim, H.K., Kim, H., Chungg, Y.C.: 20-gb/s polar RZ 4-pam transmission over 20-km SSMF using RSOA and direct detection. Photonics technology letters, Vol. 27, pp. 1116-1119, IEEE (2015)

6. Andrews L.C., Phillps, R.L.: Laser beam propagationthroughrandom media, Washington.SPIE Press.Bellingham, pp. 782 (2005)

7. Atef, M., Gaberl, W., Swoboda, R., Zimmermann, H.: An integrated optical receiver for multilevel data communication over plastic optical fiber. IEEE, (2009)

8. Kikuchi, N.: Intersymbol interference (ISI) suppression technique for optical binary and multilevel signal generation. Journal of lightwave technology, Vol. 25, pp. 2060-2068 Japan (2007)

9. Proakis, J., Digital Communications. Mc Graw Hill (2001)

10. Poteras, C.M., Mihaescu, M.C., Mocanu, M.: An optimized version of the K-means clustering. Proceedings of the 2014 Federated Conference on Computerr Science and Information Systems, Vol. 2, pp. 695-699, IEEE, Rumania (2014)

11. Teknomo, K.: K-means clustering tutorial. http://people.revoledu.com/kardi/tutorial/kMean/ (2007)

12. Yang, Y., Chen, Q., Wan, Y.: A fast near-optimum block truncation coding method using a truncated K-means algorithm and inter-block correlation. International Journal of Electronics and Communications (AEÜ) China (2010) 